\title{
How monoxenous trypanosomatids revealed hidden feeding habits of their tsetse fly hosts
}

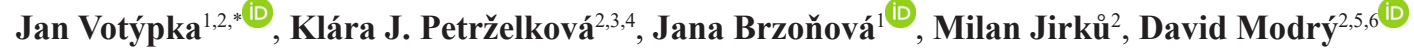 \\ and Julius Lukeš $\breve{2}^{2,7}$ (D) \\ ${ }^{1}$ Department of Parasitology, Faculty of Science, Charles University, Prague, Czech Republic; \\ ${ }^{2}$ Institute of Parasitology, Biology Centre, Czech Academy of Sciences, České Budějovice (Budweis), Czech Republic; \\ ${ }^{3}$ Institute of Vertebrate Biology, Czech Academy of Sciences, Studenec, Czech Republic; \\ ${ }^{4}$ Liberec Zoo, Liberec, Czech Republic; \\ ${ }^{5}$ Department of Botany and Zoology, Faculty of Science, Masaryk University, Brno, Czech Republic; \\ ${ }^{6}$ Department of Veterinary Sciences, Faculty of Agrobiology, Food and Natural Resources, Czech University of Life Sciences, \\ Prague, Czech Republic; \\ ${ }^{7}$ Faculty of Sciences, University of South Bohemia, České Budějovice (Budweis), Czech Republic \\ * corresponding author
}

\begin{abstract}
Tsetse flies are well-known vectors of trypanosomes pathogenic for humans and livestock. For these strictly blood-feeding viviparous flies, the host blood should be the only source of nutrients and liquids, as well as any exogenous microorganisms colonising their intestine. Here we describe the unexpected finding of several monoxenous trypanosomatids in their gut. In a total of 564 individually examined Glossina (Austenia) tabaniformis (Westwood) (436 specimens) and Glossina (Nemorhina) fuscipes fuscipes (Newstead) (128 specimens) captured in the Dzanga-Sangha Protected Areas, Central African Republic, 24 (4.3\%) individuals were infected with monoxenous trypanosomatids belonging to the genera Crithidia Léger, 1902; Kentomonas Votýpka, Yurchenko, Kostygov et Lukeš, 2014; Novymonas Kostygov et Yurchenko, 2020; Obscuromonas Votýpka et Lukeš, 2021; and Wallacemonas Kostygov et Yurchenko, 2014. Moreover, additional 20 (3.5\%) inspected tsetse flies harboured free-living bodonids affiliated with the genera Dimastigella Sandon, 1928; Neobodo Vickerman, 2004; Parabodo Skuja, 1939; and Rhynchomonas Klebs, 1892. In the context of the recently described feeding behaviour of these dipterans, we propose that they become infected while taking sugar meals and water, providing indirect evidence that blood is not their only source of food and liquids.
\end{abstract}

Keywords: Glossina, blood-feeding, adenotrophic viviparity, bodonids, Trypanosoma, infection.

Although the distribution of tsetse flies (Glossina spp.) is geographically limited to sub-Saharan countries, they are widely (in)famous as vectors of human and animal African trypanosomiases, also known as sleeping sickness and nagana, respectively (Steverding 2008, Gibson 2017). These multivoltine robust blood-feeding flies have a rather unique lifestyle, which includes particularly aggressive attacks on mammals (Stuhlmann 1907, Buxton 1955). Millions of people are estimated to be at risk of infection by the transmitted trypanosomes, with the economic losses on livestock reaching billions of US dollars per year (WHO 2012, Vreysen et al. 2013). The reduction and control of tsetse fly populations is an achievable goal and represents a very effective method for controlling trypanosomiases.
However, it requires a thorough knowledge of the biology and ecology of these flies (Scoones 2014).

Tsetse flies exhibit specialised reproductive biology, defined as adenotrophic viviparity. Similar to other members of the superfamily Hippoboscoidea, the eggs hatch within a modified uterus where the larvae are nourished on prodigious maternal milk-like secretions. Eventually, the large third instar larvae are deposited and almost immediately pupate, giving rise to adult flies a few weeks later (Leak 1998, Haines et al. 2020). Owing to the fact that the tsetse flies are viviparous, exogenous microorganisms such as bacteria, viruses, and protists shall not be able to colonise the gut of newly emerged (teneral) flies during larval development, a phenomenon otherwise common in other flies

Address for correspondence: Jan Votýpka: Department of Parasitology, Faculty of Science, Charles University, Viničná 7, CZ-128 44 Prague 2, Czech Republic. E-mail: jan.votypka@natur.cuni.cz; Julius Lukeš: Institute of Parasitology, Biology Centre, Branišovská 31, CZ-370 05 České Budějovice (Budweis), Czech Republic. E-mail: jula@paru.cas.cz 
and insects. Moreover, both sexes are strictly hematophagous, another rather unique feature among the blood-sucking flies (Buxton 1955).

Consequently, the blood, generally considered to be sterile, was supposed to be the only source of food and water throughout the life cycle of tsetse flies. If true, the engorged blood should also be the sole source of any intestinal microorganisms. However, it has to be considered that tsetse may still acquire some bacteria from other external sources, in particular being contaminated with microbes present on the skin of humans and animals during blood feeding (Wang et al. 2013). Moreover, several maternally-transmitted endosymbiotic bacteria have been described in tsetse, namely Wigglesworthia glossinidia, Sodalis glossinidius, and species of Wolbachia and Spiroplasma (see Wang et al. 2013, Doudoumis et al. 2017).

However, several recent data on the microbial communities in the tsetse gut are difficult to reconcile with the above long-term presuppositions. Focusing on the composition of gut bacterial communities of wild tsetse flies (Glossina palpalis palpalis (Robineau-Desvoidy)), Ngoune et al. (2019) documented an unexpected diversity of more than 10 bacterial genera and speculated that they may originate from external non-blood sources. Indeed, in the same organ, numerous bacteria have been detected that are commonly found in soil and water (Gaithuma et al. 2020). In addition to this indirect link, the ability of tsetse to digest sugar has been proposed (D'Costa et al. 1973) and eventually observed in a laboratory-reared colony of Glossina palpalis gambiensis (Vanderplank) by Solano et al. (2015). Consequently, it was proposed that tsetse may feed on and, in parallel, acquire bacteria from a broad range of nectar plants (Solano et al. 2015).

These new findings prompted us to revisit our previous observations, which were hard to explain in frame of the paradigm that blood is the only source of nutrition of tsetse flies. During an investigation of tsetse flies in the forest ecosystem of the Dzanga-Sangha Protected Areas, Central African Republic, we detected not only frequently transmitted members of the genus Trypanosoma Gruby, 1843, but also non-trypanosome trypanosomatids that were so far known only as parasites of insects, and bodonids with a free-living lifestyle (Votýpka et al. 2015). Originally, we have considered this 'bycatch' as possible contamination.

Although dixenous parasites of the genera Trypanosoma and Leishmania Ross, 1903 are the best-known members of the family Trypanosomatidae, the majority of parasitic kinetoplastids is constituted by monoxenous species (Kostygov et al. 2021). The latter flagellates are usually found in the midgut and hindgut of two large groups of insects - flies (Diptera) and true bugs (Heteroptera) (Lukeš et al. 2018). These protists are transmitted among insect hosts either by feeding on infected prey or fresh faeces (i.e. coprophagy) or via contaminated substrates, such as sugar meal (Maslov et al. 2013, Frolov et al. 2021). These transmission routes are permissive to non-specific infections, and the available data indicate that monoxenous trypanosomatids are able to survive and even multiply (at least for some time) in a wide range of hosts (Lukeš et al.
2018, Kostygov et al. 2021). However, it remains unclear whether the accidental hosts play any significant role in their transmission and ecology.

Most monoxenous trypanosomatids of insects are considered non-pathogenic or even commensals (but see Schaub 1994, Hamilton et al. 2015, Gómez-Moracho et al. 2020), yet they are occasionally transmitted to mammals including humans, in which they can cause infections (Dedet and Pratlong 2000, Maslov et al. 2013). Finally, these flagellates are very interesting from the evolutionary point of view, as dixenous trypanosomes and leishmanias are derived from primarily insect pathogens (Lukeš et al. 2014).

Altogether, 564 tsetse flies captured in September 2012 in the Dzanga-Sangha Protected Areas were individually screened for the presence of kinetoplastid flagellates using the nested PCR-amplified (with primer pairs S763 + S762 and TrN-F2 + TrN-R2) 18S rRNA gene (Votýpka et al. 2015). Along with the expected and in detail characterised Trypanosoma spp. (Votýpka et al. 2015), eight species of monoxenous trypanosomatids were detected in $24(4.3 \%)$ tsetse flies determined as Glossina (Austenia) tabaniformis (17 out of 436 examined, i.e. 3.9\%) and Glossina (Nemorhina) fuscipes fuscipes (7/ 128, i.e. 5.5\%).

The set of seven detected non-trypanosome trypanosomatids is composed of members of five monoxenous genera (Fig. 1). Among them, the most frequent was the genus Crithidia Léger, 1902, with two already described species and one novel typing unit (TU). In the absence of morphological data, TU serves as a proxy to species (Westenberger et al. 2004), represented by four different genotypes (Fig.1). The other four trypanosomatids belong to different genera, namely Kentomonas Votýpka, Yurchenko, Kostygov et Lukeš, 2014; Wallacemonas Kostygov et Yurchenko, 2014; and Obscuromonas Votýpka et Lukeš, 2021, within which they form novel TUs, while a member of the genus Novymonas Kostygov et Yurchenko, 2020 clearly belongs to the well-described species Novymonas esmeraldas Votýpka, Kostygov, Maslov et Lukeš, 2020. Although the diversity of these flagellates in tsetse is surprising, most of the detected genera have already been described from the African dipterans and/or heteropterans (Votýpka et al. 2012, 2019, Kostygov et al. 2016, Lukeš et al. 2021). Thus, their acquisition by tsetse flies via a sugar diet contaminated by faeces of other insect hosts is the most plausible explanation.

Flagellates belonging to the species-rich genus Crithidia have a wide host range, being found in the gut of dipteran, heteropteran and hymenopteran insects (Wallace 1966, Podlipaev 1990). The $18 \mathrm{~S}$ rDNA sequences amplified from Glossina f. fuscipes (G42) and G. tabaniformis (G141) are $100 \%$ identical with the recently described Crithidia dobrovolskii Ganyukova et Frolov, 2019, which was isolated from the rectum of the tachinid fly Lypha dubia (Fallén, 1810) captured in the Leningrad Region, Russia (Ganyukova et al. 2019). Hence, our finding significantly extends the geographic distribution of this parasite from northern Europe to sub-Saharan Africa. With more extensive sampling, such a wide geographical distribution becomes an increasingly more frequent feature of monoxenous try- 

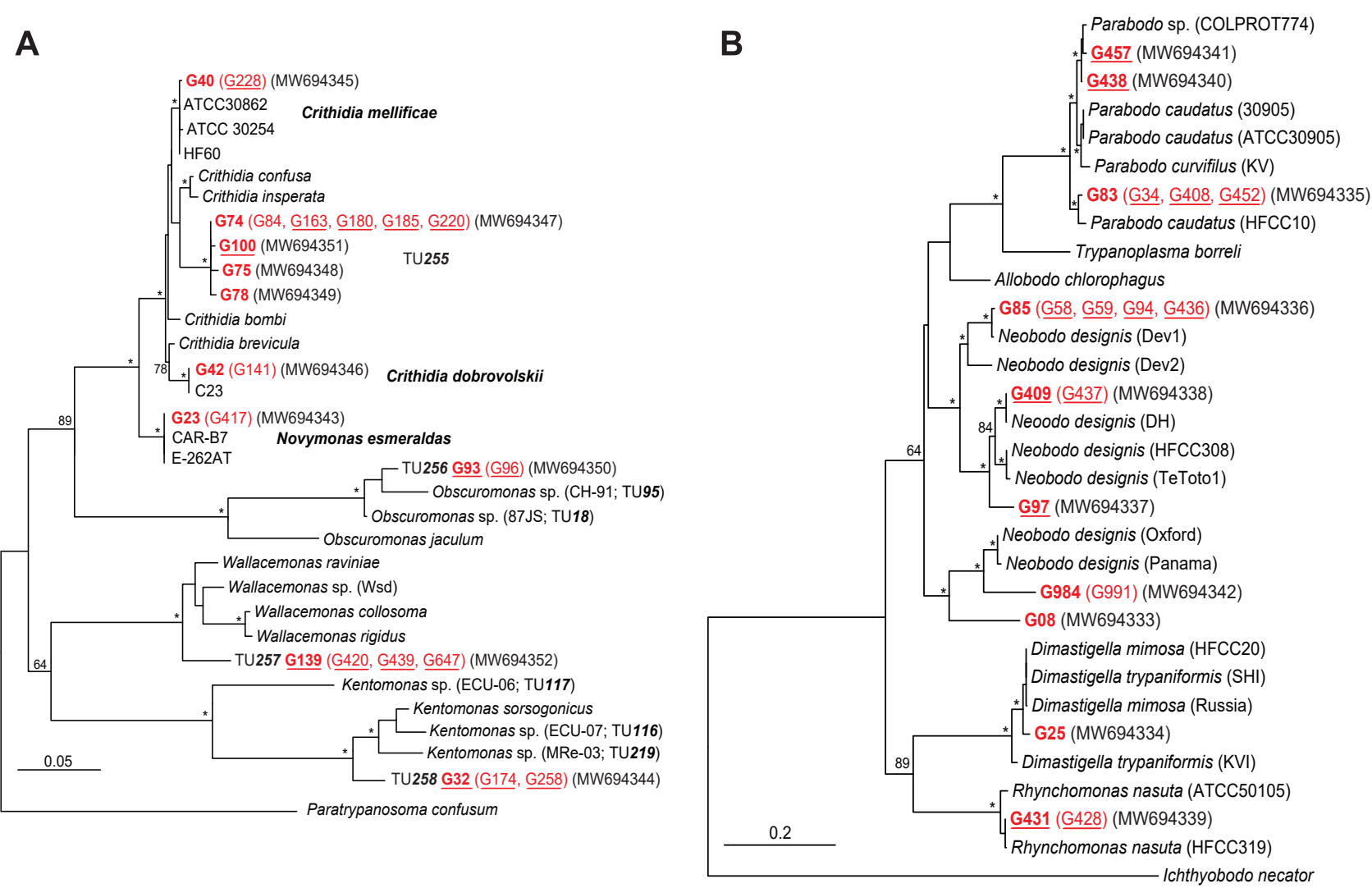

Fig. 1. Phylogenetic trees of monoxenous trypanosomatids (A) and bodonids (B) based on the 18S rRNA gene sequences and reconstructed using the Maximum likelihood method. Asterisks mark branches with maximal statistical support (bootstrap values for maximum likelihood $>90$, Bayesian posterior probabilities $>0.95$ ); the scale bar denotes the number of substitutions per site. Newly obtained sequences from tsetse flies (underlined samples are from Glossina (Austenia) tabaniformis (Westwood, 1850), others are from Glossina (Nemorhina) fuscipes fuscipes (Newstead, 1911)) captured in the Dzanga-Sangha Protected Areas, Central African Republic are in red (bold - representative sequences that have been deposited to GenBank, other samples are in parentheses).

panosomatids that have low host specificity (Maslov et al. 2013, Votýpka et al. 2020), allowing some species a cosmopolitan distribution (Lukeš et al. 2018, 2021).

The other encountered species of Crithidia, C. mellificae Langridge et McGhee, 1967, found in both investigated tsetse fly species (G40, G228), is a cosmopolitan parasite of honeybees (Apis mellifera Linnaeus, 1758) and solitary bees (Osmia spp.) (Schwarz et al. 2015, Strobl et al. 2019). This protist was also detected by PCR in the horsefly Haematopota griseicoxa Oldroyd, 1952 captured in the Central African Republic (Votýpka et al. 2019). Furthermore, 18S rDNA sequences obtained from four $G$. f. fuscipes and five G. tabaniformis are closely related to another described Crithidia species (Fig. 1A). They constitute a new TU represented in our dataset by four genotypes that differ among themselves by four to seven nucleotide substitutions.

The monospecific genus Novymonas has been described from the hindgut of the true bugs (Hemiptera) Niesthrea vincentii (Westwood, 1842) and Zelus sp. from Ecuador, and the biting midges Culicoides cf. fulvithorax (Austen, 1912) and Culicoides cf. distinctipennis Austen, 1912 from the Central African Republic and Gabon, respectively (Kostygov et al. 2016). While the question of the primary host of this unusual flagellate remains open, our finding further confirms its circulation in the sub-Saharan insects.
Another TU present in our dataset clearly belongs to the species-poor endosymbiont-containing genus Kentomonas. Its type species Kentomonas sorsogonicus Votýpka et Lukeš, 2014 was isolated from the hindgut of the brachyceran fly Sarcophaga sp. in the Philippines (Votýpka et al. 2014). A handful of other TUs affiliated with this genus originates from flies of the families Sarcophagidae (genus Ravinia Robineau-Desvoidy, 1863) and Lauxaniidae (unspecified genus) from Ecuador (Votýpka et al. 2014). Given the known host range, as well as the host specificity of the closely related genera Angomonas Souza et Corte-Real, 1991 and Strigomonas Lwoff et Lwoff, 1931 (Teixeira et al. 2011, Lukeš et al. 2018), it can be assumed that the primary hosts of Kentomonas spp. are dipteran insects. While for members of the genera Crithidia, Novymonas, and Kentomonas it was plausible to consider Diptera as their primary or at least common hosts, this question remains open for the cosmopolitan genera Wallacemonas and Obscuromonas, the latter of which produces cyst-like amastigotes. Both primarily parasitise heteropteran bugs and so far have not been found in dipteran flies (Kostygov et al. 2016, Lukeš et al. 2021), with the single exception of Wallacemonas raviniae (Votýpka et Lukeš, 2014) from the fly $R a$ vinia sp. (Diptera: Brachycera: Sarcophagidae) (Yurchenko et al. 2014). We suggest that in all the above-described cases, the detected monoxenous trypanosomatids are not 
primarily parasites of tsetse. It is more plausible that these flagellates, shown to be excreted with faeces from their natural hosts, have been acquired by a contaminative transmission that occurred in the course of the nectar-feeding behavior of tsetse.

Due to the extremely high sensitivity of nested PCR, the risk of contamination has to be considered. However, the possibility of contamination in the laboratory can be ruled out not only because of numerous controls, but also thanks to the considerably high diversity of detected trypanosomatids and bodonids. Another possible source of contamination is the surface of the tsetse fly (Ngoune et al. 2019). To eliminate this risk, the flies were thoroughly and repeatedly washed before homogenisation and DNA extraction.

Whereas we suppose that the detected monoxenous trypanosomatids originate from a sugar diet taken by flies, the possibility of accidental contamination from the skin of the host, from which tsetse took blood, cannot be rigorously excluded (Wang et al. 2013, Ngoune et al. 2019), as the animal skin inevitably harbours a range of microorganisms acquired from the environment. In addition, feeding of tabanids and tsetse regularly results in blood oozing from the host skin, attracting various flies, the faeces of which can be a source of monoxenous flagellates, such as Kentomonas spp. However, this scenario cannot explain the occurrence of Crithidia mellificae from honeybees or $\mathrm{Ob}$ scuromonas spp. parasitising heteropterans.

In addition to the trypanosomatid parasites, we have also detected five bodonid species in 20 (3.5\%) examined tsetse flies. While five TUs of the genus Neobodo Vickerman, 2004, one Rhynchomonas Klebs, 1892, and one Dimastigella Sandon, 1928 are neobodonids, three TUs of Parabodo Skuja, 1939 belong to the parabodonid clade. Curiously, these ubiquitous heterotrophic flagellates have not yet been associated with parasitism, as they are encountered in the aquatic environment (Flegontova et al. 2020). Indeed, bodonids belong to the most frequent protist groups in benthic communities, and the simplest explanation for their presence in the gut of tsetse is the acquisition by water uptake. All these genera, known for solitary and phagotrophic lifestyles, are generally abundant (Kostygov et al. 2021).

Two of the newly obtained sequences (G428 and G431) are identical to the sequence of Rhynchomonas nasuta (Stokes, 1888) (HFCC319; Acc. No. DQ207598), whereas the other two (G409 and G437) are identical to the sequence of Neobodo designis (Skuja, 1948) (DH; AF464896) (Fig. 1B). Several other sequences represented by G25, G58, G83, G438, and G457 are highly similar to, but not identical with, the already available sequences of Dimastigella mimosa Frolov, Mylnikov et Malysheva, 1997 / D. trypaniformis Sandon, 1928, N. designis, and Parabodo caudatus (Dujardin, 1841); whereas three sequences (G08, G984, and G97) constitute new branches within the genus Neobodo (Fig. 1B). Members of the environmentally less frequent genus Parabodo have been previously detected not only as aquatic phagotrophic biflagellates but also in the stool and urine samples of animals. Such tolerance may be a preadaptation of parabodonids to parasitism, which indeed seems to have originated in this group at least twice (Lukeš et al. 2014). The amplified 18S rRNA genes represent three slightly different genotypes associated with the species P. caudatus or Parabodo curvifilus (Griessmann, 1914). Since a few entomophilic bodonids have been already encountered by Lipa (1963) and bodonid DNA has been PCR-amplified from vertebrate host samples on several occasions, including the blood of homoeothermic animals (Dario et al. 2017, Szőke et al. 2017), the above-described detection of neobodonids and parabodonids is not unprecedented. However, the rarity of descriptions of bodonids from the animal samples may be skewed by the fact that as generally free-living heterotrophs, they may be considered by many as contamination rather than as a genuine component and dismissed. Although the most plausible explanation is the acquisition of bodonids with water, alternative hypotheses should also be considered, such as contamination during the feeding on (semi)aquatic animals, a behaviour observed for several tsetse species including $G$. fuscipes.

Combined, our findings of protists in the intestine of tsetse flies support previous laboratory observations of their feeding habits that are apparently more complex than just the well-known blood feeding (Solano et al. 2015). It is also worth noting that the adult tsetse flies live significantly longer than other insect vectors, which compensates for their slow rate of reproduction (Buxton 1955, Leak 1998). Such extended lifespan provides an increased chance for the infection by monoxenous trypanosomatids acquired from a contaminated sugar meal, as well as by free-living bodonids from water bodies. It is very likely that both invertebrates and vertebrates are able to host, usually for only a limited period of time, a variety of trypanosomatids and bodonids. However, since these flagellates are not expected in this wide array of hosts, when encountered, they may be dismissed as contaminations. Indeed, this was the case of tsetse flies, which are intensely studied as vectors of trypanosomes. It was only the observations of their so far unknown behaviour that prompted us to revisit our previous findings. Careful examinations for the presence of these flagellates will surely unearth possibly quite frequent, yet likely (very) mild and asymptomatic infections in insects, as well as in vertebrates including mammals.

Acknowledgments. Czech Science Foundation grant (2007186S), ERC CZ grant LL1601, and the ERD Funds of the Czech Ministry of Education (16_019/0000759) supported this work. We would like to express our gratitude to the government of the Central African Republic and the World Wildlife Fund for granting permission to conduct our research, and the Primate Habituation Programme for logistical support in the field. We also thank the field trackers and assistants. 


\section{REFERENCES}

Buxton P.A. 1955: The Natural History of Tsetse Flies: An Account of the Biology of the Genus Glossina (Diptera). H. K. Lewis \& Co., London, 739 pp.

Dario M.A., Moratelli R., Schwabl P., Jansen A.M., LLEWELLYN M.S. 2017: Small subunit ribosomal metabarcoding reveals extraordinary trypanosomatid diversity in Brazilian bats. PLoS Negl. Trop. Dis. 11: e0005790.

D’Costa M.A., Rice M.J., Latif A. 1973: Glycogen in the proventriculus of the tsetse fly. J. Insect Physiol. 19: 427-433.

Dedet J.P., Pratlong F. 2000: Leishmania, Trypanosoma and monoxenous trypanosomatids as emerging opportunistic agents. J. Eukaryot. Microbiol. 47: 37-39.

Doudoumis V., Blow F., Saridaki A., Augustinos A., Dyer N.A., Goodhead I., Solano P., Rayaisse J.-B., Takac P., Mekonnen S., Parker A.G., AbD-Alla A.M.M., Darby A., Bourtzis K., Tsiamis G. 2017: Challenging the Wigglesworthia, Sodalis, Wolbachia symbiosis dogma in tsetse flies: Spiroplasma is present in both laboratory and natural populations. Sci. Rep. 7: 4699.

Flegontova O., Flegontov P., Londoño P.A.C., Walczowsk W., Šantić D., Edgcomb V.P., Lukeš J., Horák A. 2020: Environmental determinants of the distribution of planktonic diplonemids and kinetoplastids in the oceans. Env. Microbiol. 22: 4014-4031.

Frolov A.O., Kostygov A.Y., Yurchenko V. 2021: Development of monoxenous trypanosomatids and phytomonads in insects. Trends Parasitol. 37: 538-551.

Gaithuma A., Yamagishi J., Hayashida K., Kawai N., Namangala B., Sugimoto CH. 2020: Blood meal sources and bacterial microbiome diversity in wildcaught tsetse flies. Sci. Rep. 10: 5005.

Ganyukova A.I., Malysheva M.N., Smirnov P.A., Frolov A.O. 2019: Crithidia dobrovolskii sp. n. (Kinetoplastida: Trypanosomatidae) from parasitoid fly Lypha dubia (Diptera: Tachinidae): morphology and phylogenetic position. Protistology 13 206-214.

Gibson W. 2017: Kinetoplastea. In: J.M. Archibald, A.G.B. Simpson, C.H. Slamovits (Eds.), Handbook of the Protists. Springer, Cham, pp. 1089-1138.

Gómez-Moracho T., Buendía-Abad M., Benito M., García-Palencia P., Barrios L., Bartolomé C., Maside X., Meana A., Jiménez-Antón M.D., Olías-Molero A.I. Alunda J.M., Martín-Hernández R., Higes M. 2020 Experimental evidence of harmful effects of Crithidia mellificae and Lotmaria passim on honey bees. Int. J. Parasitol. 50: 1117-1124.

Haines L.R., Vale G.A., Barreaux A.M.G., Ellstrand N.C., Hargrove J.W., English S. 2020: Big baby, little mother: tsetse flies are exceptions to the juvenile small size principle. BioEssays 42: 2000049.

Hamilton P.T., VotÝpka J., Dostálová A., Yurchenko V., Bird N.H., Lukeš J., Lemaitre B., Perlman S.J. 2015: Infection dynamics and immune response in a newly described Drosophila-trypanosomatid association. mBio 6: e01356-15.

Kostygov A.Y., Karnkowska A., VotýpKa J., Tashyreva D., Maciszewski K., Yurchenko V., Lukeš J. 2021: Euglenozoa: taxonomy, diversity and ecology, symbioses and viruses. Open Biol. 11: 200407.

Kostygov A., DobÁková E., Grybchuk-Ieremenko A., VÁhaLA D., Maslov D.A., VotÝPKA J., Lukeš J., YurChenko V. 2016: Novel trypanosomatid-bacterium association: evolution of endosymbiosis in action. mBio 7: e01985-15.

LEAK S.G.A. 1998: Tsetse Biology and Ecology: Their Role in the Epidemiology and Control of Trypanosomosis. CABI, Wallingford, $568 \mathrm{pp}$.
LiPA J.J. 1963: Infection caused by Protozoa other than Sporozoa. In: A.E. Steinhaus (Ed.), Insect Pathology. An Advanced Treatise. Academic Press, New York, pp. 335-358.

Lukeš J., Tesařová M., Yurchenko V., VotÝpka J. 2021: Characterization of a new cosmopolitan genus of trypanosomatid parasites, Obscuromonas gen. nov. (Blastocrithidiinae subfam. nov.). Eur. J. Protistol. 79: 125778.

Lukeš J., Butenko A., Hashimi H., Maslov D.A., VotýPKA J., Yurchenko V. 2018: Trypanosomatids are much more than just trypanosomes: clues from the expanded family tree. Trends Parasitol. 34: 466-480.

Lukeš J., SkalickÝ T., TÝČ J., VotÝPka J., Yurchenko V. 2014: Evolution of parasitism in kinetoplastid flagellates. Mol. Biochem. Parasitol. 195: 115-122.

Maslov D.A., Votýpka J., Yurchenko V., Lukeš J. 2013: Diversity and phylogeny of insect trypanosomatids: all that is hidden shall be revealed. Trends Parasitol. 29: 43-52.

Ngoune J.M.T, Reveillaud J., Sempere G., Nuiokou F., Melachio T.T., Aвate L., Tchiofo M.T., Geiger A. 2019: The composition and abundance of bacterial communities residing in the gut of Glossina palpalis palpalis captured in two sites of southern Cameroon. Parasit. Vectors 12: 151.

Podlipaev S.A. 1990: [Catalogue of World Fauna of Trypanosomatidae (Protozoa).] Zoologicheskii Institut AN SSSR, Leningrad, 177 pp. (In Russian.)

SCHAUB G.A. 1994: Pathogenicity of trypanosomatids on insects. Parasitol. Today 10: 463-468.

Schwarz R.S., Bauchan G.R., Murphy C.A., Ravoet J., De Graaf D.C., Evans J.D., De Graaf D.C., Evans J.D. 2015: Characterization of two species of Trypanosomatidae from the honey bee Apis mellifera: Crithidia mellificae Langridge and McGhee, 1967 and Lotmaria passim n. gen., n. sp. J. Eukaryot. Microbiol. 62: 567-583.

Scoones I. 2014: The Politics of Trypanosomiasis Control in Africa. STEPS Centre Working Paper 57, STEPS Centre, Brighton, 37 pp. Solano P., Salou E., Rayaisse J.-B., Ravel S., Gimonneau G., Traore I., Bouyer J. 2015: Do tsetse fies only feed on blood? Infect. Genet. Evol. 36: 184-189.

Steverding D. 2008: The history of African trypanosomiasis. Parasit. Vectors 1: 3

Strobl V., Yañez O., Straub L., Albrecht M., Neumann P. 2019: Trypanosomatid parasites infecting managed honeybees and wild solitary bees. Int. J. Parasitol. 49: 605-613.

Stuhlmann F. 1907: Beitrage zur Kenntnis der Tsetsefliege (Glossina fusca und Gl. tachinoides). Springer, Berlin-Heidelberg, $91 \mathrm{pp}$.

Szöke K., SÁndor A.D., Boldogh S.A., Görföl T., VotÝPKa J., Takács N., Estók P., Kováts D., Corduneanu A., MolNÁR V., KontschánAnd J., Hornok S. 2017: DNA of free-living bodonids (Euglenozoa: Kinetoplastea) in bat ectoparasites: potential relevance to the evolution of parasitic trypanosomatids. Acta Vet. Hung. 65: 531-540

Teixeira M.M.G., Borghesan T.C., Ferreira R.C., Santos M.A., Takata C.S., Campaner M., Nunes V.L., Milder R.V., De Souza W., Camargo E.P. 2011: Phylogenetic validation of the genera Angomonas and Strigomonas of trypanosomatids harboring bacterial endosymbionts with the description of new species of trypanosomatids and of proteobacterial symbionts. Protist 162: 503-524.

VotÝPKA J., Brzoňová J., JEŽEK J., ModrÝ D. 2019: Horse flies (Diptera: Tabanidae) of three West African countries: a faunistic update, barcoding analysis and trypanosome occurrence. Acta Trop. 197: 105069 . 
Votýpka J., Kment P., Yurchenko V., Lukeš J. 2020: Endangered monoxenous trypanosomatid parasites: a lesson from island biogeography. Biodivers. Conserv. 29: 3635-3667.

Votýpka J., Klepetková H., Jirků M., Kment P., Lukeš J. 2012: Phylogenetic relationships of trypanosomatids parasitizing true bugs (Insecta: Heteroptera) in sub-Saharan Africa. Int J. Parasitol. 42: 489-500.

Votýpka J., Kostygov A. Y., Kraeva N., Grybchuk-Ieremenko A., Tesařová M., Grybchuk D., Lukeš J., YurchENKo V. 2014: Kentomonas gen. g., a new genus of endosymbiont-containing trypanosomatids of Strigomonadinae subfam. n. Protist 165: 825-838.

Votýpka J., Rádrová J., SkalickÝ T., Jirků M., Jirsová D., Mihalca A.D., D’Amico G., Petrželková K.J., Modrý D., LUKEŠ J. 2015: A tsetse and tabanid fly survey of African great apes habitats reveals the presence of a novel trypanosome lineage but the absence of Trypanosoma brucei. Int. J. Parasitol. 45: 741-748.

Received 1 March 2021
Vreysen M.J.B., Seck M.T., Sall B., Bouyer J. 2013: Tsetse flies: their biology and control using area-wide integrated pest management approaches. J. Invertebr. Pathol. 112: S15-S25.

WALlaCe F.G. 1966: The trypanosomatid parasites of insects and arachnids. Exp. Parasitol. 18: 124-193.

Wang J., Weiss B.L., Aksoy S. 2013: Tsetse fly microbiota: form and function. Front. Cell Infect. Microbiol. 3: 69.

Westenberger S.J., Sturm N.R., Yanega D., Podlipaev S.A., Zeledon R., Campbell D.A., Maslov D.A. 2004: Trypanosomatid biodiversity in Costa Rica: genotyping of parasites from Heteroptera using the spliced leader RNA gene. Parasitology 129: $537-547$.

WHO 2012. Report of a WHO Meeting on Elimination of African Trypanosomiasis (Trypanosoma brucei gambiense). WHO, Geneva, $80 \mathrm{pp}$.

Yurchenko V., VotýpKa J., Tesařová M., Klepetková H., Kraeva N., JirkŮ M., LUKeš J. 2014: Ultrastructure and molecular phylogeny of four new species of monoxenous trypanosomatids from flies (Diptera: Brachycera) with redefinition of the genus Wallaceina. Folia Parasitol. 61: 97-112.

Cite this article as: Votýpka J., Petrželková K.J., Brzoňová J., Jirků M., Modrý D., Lukeš J. 2021: How monoxenous trypanosomatids revealed hidden feeding habits of their tsetse fly hosts. Folia Parasitol. 68: 019. 